\title{
Systemic lupus erythematosus (SLE) and its complications: A case study
}

\author{
Salman Azeem ${ }^{1}$, Syed Wasif Gillani' ${ }^{2 *}$, Vinci Poh $^{3}$ and Syed Azhar Syed Sulaiman ${ }^{4}$ \\ ${ }^{1}$ King Abdul Aziz Military Hospital, Tabuk, Saudi Arabia \\ ${ }^{2}$ School of Pharmacy, Monash University, Malaysia \\ ${ }^{3}$ School of Pharmacy, Monash University, Malaysia \\ ${ }^{4}$ School of Pharmaceutical Sciences, Univerisiti Sains Malaysia (USM), Malaysia
}

\begin{abstract}
Systemic Lupus Erythematosus (SLE) is a chronic, unpredictable and relapsing disease, predominantly affecting women of childbearing age. For severe cases of SLE, the usage of high dose toxic medications leads to a high risk of development of complications. The following case demonstrates the difficulty of managing SLE and the complications that follow.

Case presentation: KR is a 21 year old Indian female who was admitted for the chief complaint of bilateral leg swelling. She also presented with frothy urine and haematuria and diagnosed with acute nephritis and acute myelodysplatic syndrome with pancytopenia. To these, she was treated with frusemide, haemodialysis and prednisolone. Later on, her renal function worsened and was switched to IV methylprednisolone. She developed E.Coli pyelonephritis and bacteremia. Further development of autoimmune haemolytic anemia and thrombocytopenia necessitated the provision of haematinics. Discovery of severe hypertension lead to a multidrug antihypertensive regimen. A few days later, KR developed seizures likely caused by steroid toxicity, to which methylprednisolone was ceased. Phenytoin was administered to prevent fitting. However, due to her hypoalbuminemia and renal impairment she experienced phenytoin toxicity and developed nystagmus, to which phenytoin was temporarily ceased. She was continuously warded until significant improvement and was discharged. However her blood pressure is still uncontrolled. She was asked to return at a later date to do a renal biopsy.

Clinical evaluation: The timeliness and choice of treatment for treating the infections were questionable. Administration of IV Immunoglobulin was untimely and not using the usual regimen for autoimmune diseases. Miscalculation of plasma phenytoin concentration lead to phenytoin toxicity. Also, KR's uncontrolled blood pressure was not properly solved before discharge.
\end{abstract}

\section{Introduction}

Systemic Lupus Erythematosus (SLE) is a chronic autoimmune disease that affects multiple systems, and is most predominant among women of childbearing age. SLE is more common among Asians and black people, and among Malaysians there have been approximately 10,000 people who have been diagnosed with SLE in the past 30 years $[1,2]$. There is great variance in clinical findings, due to the potential yet not compulsory involvement of any organ system, and hence there has been much debate on the inclusion criteria for diagnosis. Currently the worldwide accepted criteria for diagnosis is the American College of Rheumatology criteria, where the presentation of at least 4 of 11 classification criteria would lead to a positive diagnosis. The eleven criteria include a malar rash, discoid rash, photosensitivity, oral ulcers, arthritis, serositis, renal manifestations, neurological manifestations, haematological manifestations, immunological manifestations or a positive antinuclear antibody (ANA) result. However now there is a revised and more favoured version created by the Systemic Lupus International Collaborating Clinics (SLICC), which includes 11 clinical criteria and 6 immunologic criteria, and a presentation of at least 4 of these 17 criteria, with at least one coming from each group, would lead to a positive diagnosis. The 11 clinical criteria are: acute cutaneous lupus, chronic cutaneous lupus, non-scarring alopecia, nasal or oral ulcers, joint disease, serositis, renal manifestations, neurologic manifestations, haemolytic anemia, leukopenia or lymphopenia and thrombocytopenia. The 6 immunologic criteria are: above range ANA levels, above range anti-dsDNA levels, positive antiphospholipid antibodies, low complement and a direct Coombs test $[1,3]$.

SLE is a relapsing and unpredictable disease. Long term prognosis tends to be good if the disease is controlled during the initial acute phase, hence it is of utmost importance for the patient to not delay in seeking treatment and for the healthcare team to identify the most effective treatment as soon as possible. Pharmacological treatment varies depending on the severity of the disease as well as the organ systems involved. Mild presentation of symptoms may only require the use of NSAIDS or antimalarials, while more severe disease may require corticosteroids or immunosuppressants [1]. Unfortunately with more toxic medications comes a higher mortality risk due to a higher risk of complications, and adverse effects due to high doses can

Correspondence to: Syed Wasif Gillani, School of Pharmacy, Monash University Malaysia, PO box 46150, Bandar Sunway, Selangor, Malaysia, Tel: +60174203027; E-mail: wasifgillani@gmail.com

Key words: systemic lupus erythematosus, complications, prednisolone, renal, haemotological, neurological

Received: May 13, 2015; Accepted: June 23, 2015; Published: June 26, 2015 
make interpretations of clinical presentations or clinical responses complicated [3].

It is therefore important that the management of SLE patients require a healthcare team of multiple disciplines and professions, with close monitoring and control to prevent the worsening of the disease or the development of complications. This case study presents a case of an SLE patient who has developed complications during her course of treatment.

\section{Case presentation}

$\mathrm{KR}$ was a $70 \mathrm{~kg}$, 21 year old Indian femalewho was admitted on 21-09-2003, with a chief complaint of bilateral leg swelling for 2 days. She also presented with frothy urine and haematuria, to which she was treated with frusemide. KR was diagnosed with acute nephritis and was hence started on haemodialysis treatment. In addition to that, she was also diagnosed with acute myelodysplatic syndrome with pancytopenia, to which prednisolone treatment was given.

On 25-09-2003 the patient was diagnosed with SLE. It was discovered that her SLE has been misdiagnosed as leukemia back in 2001. Due to worsening renal failure she has been placed on IV methylprednisolone. She also contracted E.coli pyelonephritis which was sensitive to ceftazidime and ampicillin+sulbactam. She also has bacteremia as the microbiology test revealed E.coli in her blood. Further development of autoimmune haemolytic anemia and thrombocytopenia necessitated the provision of haematinics. Diagnosis of severe hypertension lead to treatment involving captopril, nifedipine, metoprolol, atenolol, prazosin, diltiazem and frusemide.

On 29-09-2003 the patient's condition took a turn for the worst as she developed seizures secondary to either cerebral lupus or steroid toxicity, hence she was taken off steroids. A CT scan ruled out intracranial haemorrhage. Despite being on a total of $1250 \mathrm{mg} /$ day of phenytoin, the patient continued fitting and only ceased after 2 days. Due to phenytoin toxicity the patient developed secondary nystagmus. It was noted that the patient was restarted on hydrocortisone on the $30^{\text {th }}$, despite there being no record of her being started on it.

KR's past medical history included the misdiagnosis of leukemia in 2001, and a history of myelodysplastic syndrome. She did not have hypertension prior to admission and had never had symptoms of anemia or bleeding. As for family history, her grandmother had hypertension and her mother had thyrotoxicosis. The patient is currently staying with her family and is not studying nor working.

A medical history interview revealed that other than the complaints mentioned above, KR has also experienced headaches, dizziness, fainting, malenic stools and sleeping problems. She does not have any known food or drug allergies, and does not consume alcohol nor smoke. KR is non-compliant to her medications. Her medication history includes Ferrous Sulphate tablets $200 \mathrm{mg}$ once a day (OD), Folate tablets $5 \mathrm{mg}$ OD, Prednisolone tablets every other day (EOD) and Vitamin B Complex tablets 5mg OD, all starting from 2001 onwards. Her diet is imbalanced and she does not engage in physical exercise.

Laboratory data collected from 24/9 till 19/10 shows that her blood urea nitrogen (BUN) and creatinine levels are too high, and hence creatinine clearance $(\mathrm{CrCL})$ is too low. Her daily fluid balance steadily decreased, which is good due to her fluid retention. Haematological wise, her haematocrit levels are too high while all other components are too low. Only white blood cells (WBC) remain within normal levels.
Liver function tests show total protein and albumin levels are too low. Coagulation profiles were taken on $14 / 10$ and revealed that INR is too low and activated partial prothrombin time (APPT) is too long.

The list of diagnoses, differential diagnoses, acute and chronic medical problems of KR are as follows:

1. Acute myelodysplatic syndrome with pancytopenia

2. Acute pyelonephritis with acute glomerulonephritis

3. Bacteremia

4. Autoimmune haemolytic anemia \& thrombocytopenia

5. SLE

6. Hypertension

7. Seizure

Table 1 shows her medication regimen during her ward stay, inclusive of medication administration times.

Table 2 shows her progress from 13-10-2003 till 20-10-2003.

Table 3 includes laboratory data taken from 24/9 till 19/10.

Table 1. Inpatient medication record.

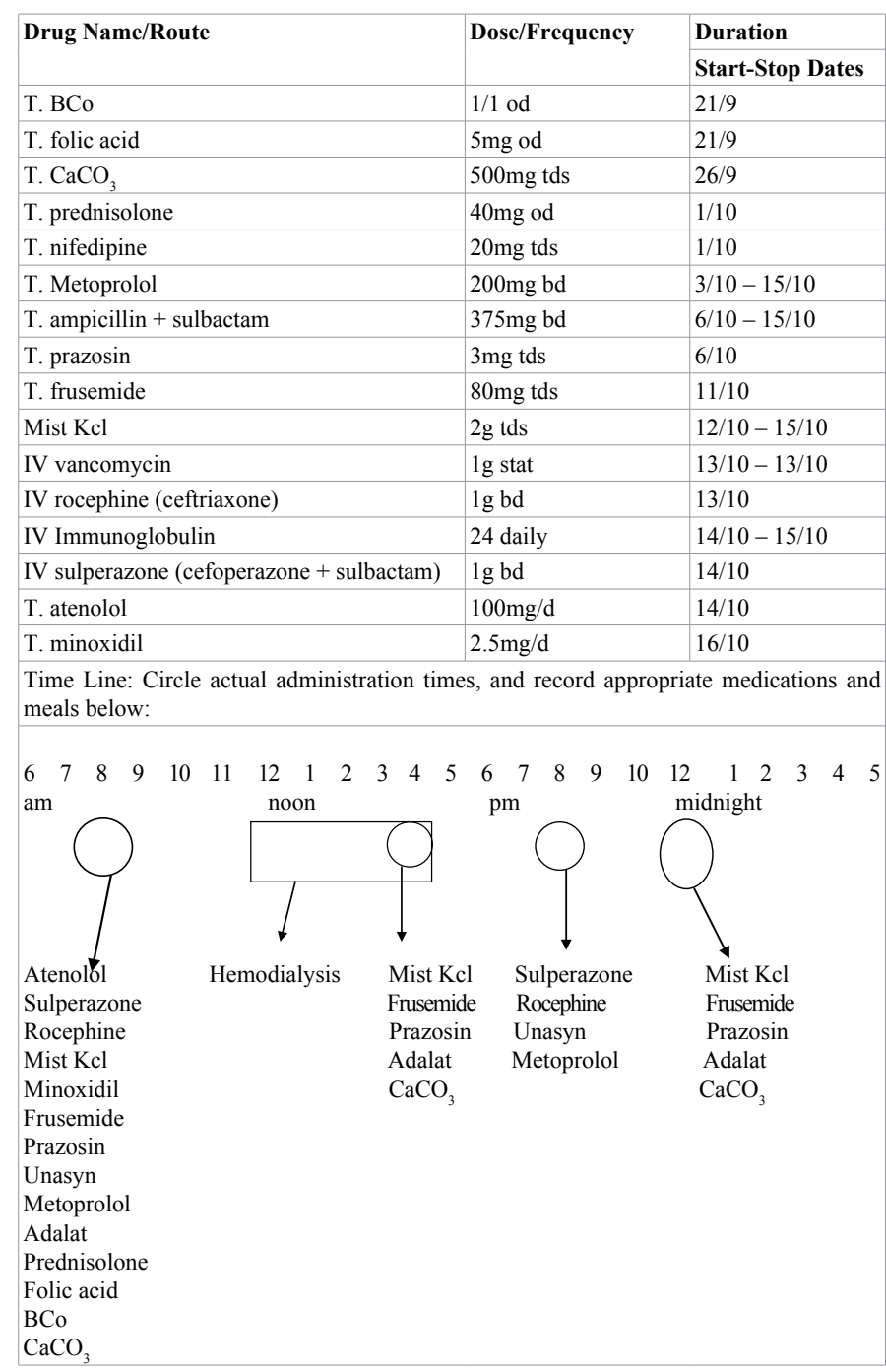


Table 2. Inpatient progress notes.

\begin{tabular}{|c|c|c|c|c|c|c|c|c|c|}
\hline Date & & $13 / 10$ & $14 / 10$ & $15 / 10$ & $16 / 10$ & $17 / 10$ & $18 / 10$ & $19 / 10$ & $20 / 10$ \\
\hline \multirow[t]{5}{*}{ General } & & Pale + & $\begin{array}{l}\text { Comfortable; } \\
\text { no cough }\end{array}$ & Afebrile & $\begin{array}{l}{ }^{\circ} \mathrm{c} / \mathrm{o} \text {, fever, } \\
\text { bleeding }\end{array}$ & $\begin{array}{l}\text { Comfortable; } \\
\text { tolerating well } \\
\quad \text { orally }\end{array}$ & $\begin{array}{c}\text { Keeping well; no new } \\
\text { complaints; no new } \\
\text { bruise }\end{array}$ & $\begin{array}{c}\text { Comfortable; tolerating } \\
\text { well orally; no nausea or } \\
\text { vomiting }\end{array}$ & $\begin{array}{l}\text { Comfortable; patient } \\
\text { discharged and asked to } \\
\text { come the following week } \\
\text { for her renal biopsy. }\end{array}$ \\
\hline & $\mathrm{BP}$ & $135 / 100$ & $190 / 130$ & $170 / 132$ & $182 / 119$ & $150 / 106$ & $130 / 80$ & $160 / 110$ & $180 / 90$ \\
\hline & PR & 88 & 75 & 72 & 74 & - & 80 & - & 74 \\
\hline & $\mathrm{RR}$ & - & - & - & - & - & - & - & - \\
\hline & $\mathrm{T}$ & 38.3 & Afebrile & 36.5 & 37.5 & Afebrile & Afebrile & Afebrile & Afebrile \\
\hline Lungs & & Crepts right base & Clear & & Clear & Clear & Clear & Clear & \\
\hline EXT & & Pedal edema + & & & & & & & \\
\hline
\end{tabular}

$\mathrm{BP}=$ blood pressure $(\mathrm{mmHg}) ; \mathrm{PR}=$ pulse rate $($ beats $/ \mathrm{min}) ; \mathrm{RR}=$ respiratory rate (breaths $/ \mathrm{min}) ; \mathrm{T}=$ temperature $\left({ }^{\circ} \mathrm{C}\right)$

Table 3. Laboratory data.

\begin{tabular}{|c|c|c|c|c|c|c|c|c|c|}
\hline & NORMAL & $24 / 9$ & $1 / 10$ & $13 / 10$ & $14 / 10$ & $15 / 10$ & $17 / 10$ & $18 / 10$ & $19 / 10$ \\
\hline \multicolumn{10}{|l|}{$\begin{array}{l}\text { Blood } \\
\text { Chemistry }\end{array}$} \\
\hline $\mathrm{Na}+$ & $135-145 \mathrm{mmol} / \mathrm{L}$ & 137 & 135 & & 131 & 138 & & & \\
\hline $\mathrm{K}+$ & $3.5-5.0 \mathrm{mmol} / \mathrm{L}$ & 4.3 & 3.5 & & 4.2 & 3.3 & & & \\
\hline BUN & $1.7-8.3 \mathrm{mmol} / \mathrm{L}$ & 20.2 & 24 & & 9.5 & 6.5 & & & \\
\hline Creatinine & $57-130 \mathrm{mmol} / \mathrm{L}$ & 328 & 390 & & 399 & 287 & & & \\
\hline $\mathrm{CrCl}$ & $75-125 \mathrm{ml} / \mathrm{min}$ & 26.7 & 22.47 & & 22.0 & 30.5 & & & \\
\hline \multicolumn{10}{|l|}{ Fluid } \\
\hline Input & & & & 700 & 620 & 600 & 500 & 800 & 850 \\
\hline Output & & & & 170 & 500 & 400 & 400 & 800 & 1000 \\
\hline Balance & $800-1200 \mathrm{ml}$ & & & 530 & 120 & 200 & 100 & - & -150 \\
\hline \multicolumn{10}{|l|}{ Hematology } \\
\hline Het & $0.36-0.46 \mathrm{I}$ & 17.5 & 26.5 & & & 29.3 & & & \\
\hline $\mathrm{HgB}$ & $12-16 \mathrm{~g} / \mathrm{dl}$ & 5.3 & 8.6 & & & 9.8 & & & \\
\hline WBC & $4.5-11 \times 10^{9} / \mathrm{L}$ & 5.2 & 5.0 & & & 5.8 & & & \\
\hline RBC & $4.7-6.1 \times 10^{12} / \mathrm{L}$ & 2.2 & 3.43 & & & 3.71 & & & \\
\hline $\mathrm{MCV}$ & $80-94$ & 24.3 & 77.3 & & & 78.8 & & & \\
\hline $\mathrm{MCH}$ & $27-31$ & 30.3 & 25.2 & & & 26.3 & & & \\
\hline $\mathrm{MCHC}$ & $330-370$ & 19.6 & 32.6 & & & 33.3 & & & \\
\hline PLT & $130-400 \times 10^{3} / \mu \mathrm{ml}$ & 91.1 & 91 & & & 108 & & & \\
\hline \multicolumn{10}{|l|}{ Liver Ft } \\
\hline Total Protein & $66-87 \mathrm{~g} / \mathrm{L}$ & 60 & & & & & & & \\
\hline Albumin & $38-51 \mathrm{~g} / \mathrm{L}$ & 25 & & & & & & & \\
\hline Globulin & $20-36$ & 35 & & & & & & & \\
\hline Tot bilirubin & $0-24 \mathrm{~mol} / \mathrm{L}$ & 7 & & & & & & & \\
\hline ALT & $0-42 \mathrm{U} / \mathrm{L}$ & 10 & & & & & & & \\
\hline ALP & $34-104 \mathrm{U} / \mathrm{L}$ & & & & & & & & \\
\hline PT & $10.7-13.7 \mathrm{sec}$ & 13.3 & & & 10.8 & & & & \\
\hline INR & $1.0-1.24$ & 1.1 & & & 0.9 & & & & \\
\hline APPT & $25-40 \mathrm{sec}$ & 32.2 & & & 70.6 & & & & \\
\hline
\end{tabular}

Highlighted in red are values that do not fall within recommended range

Table 4 shows results of microbiology tests done from 25/9 till $13 / 10$

\section{Clinical evaluation}

\section{Disease presentation and progression}

KR's overall SLE disease progression from admission to discharge involved renal, haematological and neurological manifestations.

Pancytopenia is a well described haematological manifestation in this disease. The significant blood cell destruction necessitated high dose pulse prednisolone treatment. Intravenous immunoglobulin (IV $\mathrm{Ig}$ ) is also recommended for the removal of destructive antibodies, however IV Ig was only administered to KR from 14/10 till 15/10, about
3 weeks after admission, and only for 2 days, despite the laboratory data that shows that while there was some degree of improvement, KR's blood component levels have yet to attain the normal ranges. IV Ig is normally given at a dose of $0.4 \mathrm{~g} / \mathrm{kg} /$ day for a duration of 5 days [4]. KR, being $70 \mathrm{~kg}$ in weight, ought to receive $28 \mathrm{~g} /$ day. Instead she received $24 \mathrm{~g} /$ day. However, this could be due to a fluctuation in the patient's weight, as while the patient was admitted with a weight of 70 $\mathrm{kg}$, control of her fluid retention may render her lighter in weight by the time they administered IV Ig.

Severely reduced renal function due to acute nephritis required haemodialysis to aid in blood filtration. This severe renal impairment may be the major contributor to the fluid retention that was the chief complaint during admission. Subsequent renal failure over 
Table 4. Microbiology tests results.

\begin{tabular}{|c|c|c|c|c|}
\hline Source & Date & Results & Sensitive to & Resistant to \\
\hline Blood & $25 / 9$ & E. coli & $\begin{array}{c}\text { Ampicilin; Ampi/sulbactam ;Amoxi/Clav ; Cotrimoxazole ;Genta, Ami, } \\
\text { Netil, Cefuroxamine; Cefoperazone ; Cefuroxime ; } \\
\text { Ceftazidime ; Ceftriaxone; Ciprofloxacin }\end{array}$ & \\
\hline Throat swab & $25 / 9$ & Normal & & \\
\hline Urine & $1 / 10$ & No significant bacteriuria & & \\
\hline Blood (arterial) & $13 / 10$ & No growth & & \\
\hline Blood (peripheral) & $13 / 10$ & No growth & & \\
\hline Blood (venous) & $13 / 10$ & No growth & & \\
\hline
\end{tabular}

the next few days necessitated the switch from oral prednisolone to IV methylprednisolone. The use of high dose immunosuppressing corticosteroids lead to the development of pyelonephritis by E.coli, which also lead to bacteremia by the same organism. The use of corticosteroids also lead to the presentation of an abnormal liver function test. These were mentioned in the eTG as common adverse effects that comes with high dose immunosuppressant therapy that may complicate clinical presentation and response interpretation [3]. Other than these, prednisolone toxicity may also lead to hypertension and fluid retention, which may partially explain why KR's blood pressure throughout her stay has been uncontrolled. It may also be possible that the water retaining adverse effect of prednisolone may have delayed the removal of excess fluids from her body [5].

The seizures that KR experienced from 29/9 were either from cerebral lupus or steroid toxicity. The chances of the cause being steroid toxicity is more likely as she only developed the seizures after a course of high dose prednisolone and later methylprednisolone; seizures are among the more serious side effects of these corticosteroids [6]. Thus, methylprednisolone was temporarily ceased and phenytoin was given to stop the seizures. Unfortunately there has been neglect in the dosing adjustments for the patient's hypoalbuminemia and renal failure, and hence the amount of free plasma fraction is higher than average and the half-life of phenytoin in the body is extended, leading to toxicity [7]. The pharmacist involved has detected and rectified this error by correcting the actual plasma concentration and suggesting to withhold phenytoin for 3 days and restarting on 3/10.

Inpatient progress notes from $13 / 10$ to $20 / 10$ revealed that KR was steadily recovering and the fluid balance is steadily being restored, with the symptoms of fluid retention being absent from 14/10 onwards. Laboratory data showed a steady improvement in blood cell component and electrolyte levels as well. The only remaining area of concern was her high, uncontrolled blood pressure. There seems to be no pattern of progress as the values fluctuate greatly from day to day. Despite this, the patient has been discharged. The patient's hypertensive treatment regimen ought to be addressed completely before discharge as aggressive blood pressure control is crucial in improving renal outcome [3].

\section{Drug choice}

KR's E.coli pyelonephritis is sensitive to ceftazidime and ampicillin+sulbactam. According to the National Antibiotic Guidelines, for acute complicated pyelonephritis, if infected by Enterococci, the preferred treatment is ampicillin 500mg IV every 6 hours plus gentamicin $5 \mathrm{mg} / \mathrm{kg}$ (70 kg=350 mg) IV every 24 hours for 2 weeks duration. Alternative treatments may be either third generation cephalosporins, $\beta$-lactam/ $\beta$-lactamase inhibitors, piperacillin/ tazobactam or ciprofloxacin [8].Oddly, despite being diagnosed with pyelonephritis as well as bacteremia on $25 / 9$, no antibiotic was given until $6 / 10$, in which she was given ampicillin+sulbactam. On $13 / 10$, despite her microbiology tests showing negative results on bacterial growth, KR was given a $1 \mathrm{~g}$ IV stat dose of vancomycin and IV ceftriaxone $1 \mathrm{~g}$ twice daily until discharge. IV sulperazone (cefoperazone + sulbactam) $1 \mathrm{~g}$ twice daily was added to the regimen on 14/10 until discharge. The antibiotic regimen is questionable and potentially dangerous to the patient's health.

For the patient's hypertension, nifedipine (calcium channel blocker) was started on $1 / 10$. Metoprolol (beta-blocker) was added on $3 / 10$ and ceased on $15 / 10$, and on $6 / 10$, prazosin (alpha-blocker) was added. Frusemide (loop diuretic) was added on $11 / 10$, and on 14/10 another beta-blocker, atenolol was added. Finally minoxidil (peripheral vasodilator) was added into the regimen on 16/10. Despite the continuous additions of anti-hypertensive medication, there seems to be no continuous improvement in KR's blood pressure control. According to the eTG, first line therapy for hypertensive patients with kidney impairment are angiotensin converting enzyme inhibitors (ACEI) or angiotensin II receptor blockers (ARB), due to their ability to not only reduce proteinuria, but to also slow the decline of kidney function. Calcium channel blockers are also a good choice as they also slow the progress of kidney failure. Loop diuretics are recommended for those with highly reduced kidney function. Care should be taken when prescribing renally excreted beta blockers due to their deleterious effects on potassium and lipid levels. Alpha blockers also have limited usefulness due to initial orthostatic hypotension, though occasionally prove to be a useful adjunct. Minoxidil is a potent vasodilator that can be extremely effective in certain individuals that have both kidney impairment and severe hypertension, but comes with the adverse effects of tachycardia and sodium retention [9]. With these in mind, the next step to control KR's hypertension may be to try an ACEI or ARB. Care should be taken to not give them together with a diuretic and an NSAID to prevent the 'triple whammy' effect. Also, it should be evaluated whether co-administration of other drugs have affected the efficacy of the anti-hypertensives. Additionally, it may be suggested that the patient practice fluid restriction.

\section{Drug-drug interactions}

Below are the potential drug-drug interactions between the drugs administered during KR's ward stay [10]:

\section{Ampicillin + Atenolol}

Co-administration may lead to decreased plasma concentrations of atenolol due to impaired gastrointestinal absorption due to ampicillin. It is suggested to adjust the dosing intervals to avoid co-administration of these two drugs, or to make ampicillin dosing smaller and more frequent. 


\section{Nifedipine + Calcium carbonate}

Calcium carbonate, a calcium product, can saturate calcium channels and hence reduce the effectiveness of nifedipine. Monitoring is needed to determine if nifedipine is working sufficiently.

\section{Beta-blockers + Calcium carbonate}

Calcium salts can decrease oral bioavailability of beta-blockers, though the exact mechanism is unknown. It is suggested to separate administration times.

\section{Beta-blockers + Prazosin}

Other than additive hypotensive effects, the combination can lead to an increased risk or severity of first dose effects of prazosin, such as syncope and postural hypotension. The reflex tachycardia that arises due to postural hypotension can be blunted by beta-blockers. Close monitoring is advised, and to start the dosages small and titre accordingly. Prazosin is advised to be taken at bedtime to reduce orthostatic effect occurrences.

\section{Prednisolone + antihypertensive medication}

Corticosteroids can cause sodium and fluid retention and hence antagonize antihypertensive effects. For those on high dose or prolonged corticosteroid therapy, such as KR, body weight, electrolyte levels and blood pressure need to be regularly monitored, and the antihypertensive medication doses may require adjustment.

\section{Nifedipine + Prazosin}

There may be potential additive hypotensive effects and increase the likelihood of postural hypotension. Close monitoring is required, particularly during initial treatment.

\section{Frusemide + beta-blockers}

Some patients are at an increased risk of developing hypertriglyceridemia and hyperglycemia with this combination, particularly diabetics. Blood glucose, blood pressure and serum potassium levels need to be monitored when these medications are coadministered.

\section{Nifedipine + beta-blockers}

Potential for additive reductions in cardiac contractility, cardiac conduction and heart rate. Haemodynamic response and tolerance of the patient should be monitored.

\section{Cephalosporins + Frusemide}

Frusemide can possibly potentiate nephrotoxicity of cephalosporins. Close monitoring of renal function is recommended, particularly since KR has pre-existing renal impairment.

\section{Frusemide + Prednisolone}

There is an increased risk of hypokalemia in the combination of potassium-depleting medication and corticosteroids. Close monitoring is required.

\section{Prednisolone + Calcium Carbonate}

Calcium carbonate exerts acid-neutralizing effects and can hence impair prednisolone absorption. However, there is no particular intervention needed for the co-administration of both drugs.

\section{Conclusion}

SLE is an exceedingly complicated disease with its involvement of multiple organ systems, and when severe requires high dose drug regimens that can easily complicate the presentation and clinical response. Thus, it is of utmost importance for the involvement of professionals from different fields, depending on the organ systems affected, to form a multidisciplinary team. Fast and accurate information flow is crucial among professionals working together in order for the patient to have a good prognosis. There needs to be constant monitoring and evaluation of therapeutic regimens of the patient along with their response, due to the complicated and severe nature of the disease.

\section{References}

1. Merck Manuals. Systemic Lupus Erythematosus (SLE) 2013.

2. Malaysian SLE Association. What is SLE?

3. eTGComplete. Systemic Lupus Erythematosus.

4. Kaveri SV, Dietrich G, Hurez V, Kazatchkine MD (1991) Intravenous immunoglobulins (IVIg) in the treatment of autoimmune diseases. Clin Exp Immunol 86: 192-198. [Crossref]

5. American College of Rheumatology (1999) Guidelines for Referral and Management of Systemic Lupus Erythematosus in Adults. USA: Lippincott-Raven Publishers.

6. Micromedex (2015) In: Truven Health Analytics, editor. Greenwood Village, Colorado.

7. Keller F, Maiga M, Neumayer HH, Lode H, Distler A (1984) Pharmacokinetic effects of altered plasma protein binding of drugs in renal disease. Eur J Drug Metab Pharmacokinet 9: 275-282. [Crossref]

8. (2008) National Antibiotic Guideline 2008.

9. eTGComplete. Elevated Blood Pressure in Patients with Kidney Disease.

10. Drugs.com. Drug Interactions Checker 2015.

Copyright: $\odot 2015$ Loudjedi S. This is an open-access article distributed under the terms of the Creative Commons Attribution License, which permits unrestricted use, distribution, and reproduction in any medium, provided the original author and source are credited. 